\title{
Vortioxetine: Clinical Pharmacokinetics and Drug Interactions
}

\author{
Grace Chen $^{1} \cdot$ Astrid-Maria Højer $^{2} \cdot$ Johan Areberg $^{2} \cdot$ George Nomikos $^{3}$
}

Published online: 30 November 2017

(c) The Author(s) 2017. This article is an open access publication

\begin{abstract}
Vortioxetine is a novel antidepressant with multimodal activity currently approved for the treatment of major depressive disorder. Vortioxetine is orally administered once daily at 5- to 20-mg doses. The pharmacokinetics of vortioxetine are linear and dose proportional, with a mean terminal half-life of approximately $66 \mathrm{~h}$ and steady-state plasma concentrations generally achieved within 2 weeks of dosing. The mean absolute oral bioavailability of vortioxetine is $75 \%$. No food effect on pharmacokinetics was observed. Vortioxetine is metabolized by cytochrome P450 enzymes and subsequently by uridine diphosphate glucuronosyltransferase. The major metabolite is pharmacologically inactive, and the minor pharmacologically active metabolite is not expected to cross the blood-brain barrier, making the parent compound primarily responsible for in-vivo activity. No clinically relevant differences were observed in vortioxetine exposure by sex, age, race, body size, and renal or hepatic function. Dose adjustment is only recommended for cytochrome P450 2D6 poor metabolizers based on polymorphism of the cytochrome P450 enzymes involved.
\end{abstract}

\section{Electronic supplementary material The online version of this} article (https://doi.org/10.1007/s40262-017-0612-7) contains supplementary material, which is available to authorized users.

Grace Chen

grace.chen@takeda.com

1 Department of Quantitative Clinical Pharmacology, Takeda Development Center Americas, Inc., One Takeda Parkway, Deerfield, IL 60015, USA

2 Clinical and Quantitative Pharmacology, H. Lundbeck A/S, Copenhagen-Valby, Denmark

3 Department of Clinical Science, Takeda Development Center Americas, Inc., Deerfield, IL, USA
Similarly, except for bupropion, a strong cytochrome P450 2D6 inhibitor, and rifampin, a broad cytochrome P450 inducer, co-administration of other drugs evaluated did not affect the vortioxetine exposure or safety profile in any clinically meaningful way. Pharmacodynamic studies demonstrated that vortioxetine achieved high levels of serotonin transporter occupancy in relevant brain areas, affected neurotransmitter levels in the cerebrospinal fluid, and modified abnormal resting state networks in the brain over the therapeutic dose range. Overall, vortioxetine can be administered in most populations studied to date without major dose adjustments; however, dose adjustments should be considered on a patient-by-patient basis.

\section{Key Points}

Vortioxetine is an antidepressant with multimodal activity currently approved for the treatment of major depressive disorder at a dosage of $5-20 \mathrm{mg} /$ day.

Vortioxetine has a favorable pharmacokinetic profile with dose-proportional and linear exposure, moderate oral bioavailability $(75 \%$; independent of food), extensive tissue distribution (steady-state volume of distribution of approximately $2600 \mathrm{~L}$ ), and a long elimination half-life (66 h).

Concomitant therapy is generally well tolerated and dosage adjustments may be required when vortioxetine is co-administered with bupropion or rifampin. 


\section{Mechanism of Action and Therapeutic Use}

Vortioxetine (Trintellix ${ }^{\circledR}$ in the USA and Canada, Brintellix ${ }^{\circledR}$ worldwide) is currently approved for the treatment of major depressive disorder (MDD) [1]. Several therapeutic agents are currently used as first-line therapy for MDD, including selective serotonin [5-hydroxytryptamine (5-HT)] reuptake inhibitors and serotonin norepinephrine reuptake inhibitors [2]. Compared with other therapeutic agents for MDD, vortioxetine has a unique mechanism of action with a distinct clinical profile, which may prove effective as an alternative first-line agent or subsequent therapy for patients with documented failure of other antidepressants [3-7].

Vortioxetine is a multimodal antidepressant with two different types of pharmacologic targets: serotonin receptors and transporters. In vitro, vortioxetine acts as a $5-\mathrm{HT}_{1 \mathrm{~A}}$ receptor agonist, $5-\mathrm{HT}_{3}, 5-\mathrm{HT}_{7}$, and $5-\mathrm{HT}_{1 \mathrm{D}}$ receptor antagonist, 5- $\mathrm{HT}_{1 \mathrm{~B}}$ receptor partial agonist, and inhibitor of the 5-HT transporter (SERT) [8]. Preclinical studies suggest that vortioxetine may exert its antidepressant activity by modulating neurotransmission in multiple systems, including serotonin, norepinephrine, dopamine, acetylcholine, histamine, glutamate, and gamma-aminobutyric acid systems [9-14]. Thus, interaction between vortioxetine and its targets may regulate complex neural networks involved in the mood and cognition-related disturbances that characterize MDD [9].

Vortioxetine (1-[2-(2,4-dimethyl-phenylsulfanyl)-phenyl]-piperazine, hydrobromide) has a molecular weight of $379.36 \mathrm{~g} / \mathrm{mol}$ [1] and a distribution coefficient (octanol/ water) ranging from $2.2(\mathrm{pH} 3)$ to $4.7\left(\mathrm{pH} \mathrm{11)}\right.$ at $25^{\circ} \mathrm{C}$ (data on file). In both acute treatment and long-term maintenance trials in patients with MDD, vortioxetine has demonstrated antidepressant efficacy and a favorable safety profile with therapeutic doses ranging from 5 to $20 \mathrm{mg}$ administered once daily [15-20]. Vortioxetine has linear and dose-proportional pharmacokinetics, with steady-state plasma concentrations generally reached within 2 weeks of once-daily dosing [21].
Compiled information on the pharmacokinetic (PK) and pharmacodynamic (PD) profile of vortioxetine from a comprehensive clinical program of more than 10 years will enable healthcare providers to better understand its drugdrug interaction potential, and whether dose adjustments are appropriate in special populations. This review summarizes phase I PK and PD data and highlights their implications in clinical practice.

\section{Clinical Pharmacokinetics}

Clinical PK parameters of vortioxetine have been evaluated in healthy study participants in 31 clinical pharmacology studies from USA, Europe, and Japan, encompassing over 1100 healthy young and elderly male and female volunteers and including individuals with hepatic and renal impairment [22]. In addition, a phase I population-PK meta-analysis of 26 studies analyzing a total of 21,758 samples for vortioxetine plasma concentrations from 887 participants who received single doses ranging from 2.5 to $75 \mathrm{mg}$ or multiple doses of 2.5-60 mg was performed [23].

\subsection{Absorption}

Vortioxetine is well absorbed orally, with an estimated minimal lag time (amount of time taken for a drug to be detectable systemically [24]) of $0.781 \mathrm{~h} \mathrm{[23].} \mathrm{The} \mathrm{maximal}$ plasma vortioxetine concentration $\left(C_{\max }\right)$ after single or multiple dosing is reached within $7-11 \mathrm{~h}$ post-dose (time to reach maximum observed plasma concentration) (Tables 1 , 2). Steady-state mean $C_{\max }$ values were 9,18 , and $33 \mathrm{ng} /$ $\mathrm{mL}$ following multiple doses of 5,10 , or $20 \mathrm{mg}$, respectively (Table 2).

Vortioxetine was shown to be a poor substrate of the P-glycoprotein (Pgp) transporter in vitro [25]. When coadministered with ketoconazole, a Pgp inhibitor, area under the curve from time 0 to time of last quantifiable concentration $\left[\mathrm{AUC}_{(0-\mathrm{t})}\right]$ and $C_{\max }$ values for vortioxetine

Table 1 Pooled non-compartmental pharmacokinetic parameters of vortioxetine following a single oral dose ${ }^{\mathrm{a}}$

\begin{tabular}{|c|c|c|c|c|c|c|c|c|c|}
\hline $\begin{array}{l}\text { Dose } \\
(\mathrm{mg})\end{array}$ & $\begin{array}{l}\text { Subjects } \\
(n)\end{array}$ & $\begin{array}{l}\text { Obs } \\
(n)\end{array}$ & $\begin{array}{l}\mathrm{AUC}_{(0-\mathrm{inf})}(\mathrm{ng} \cdot \mathrm{h} / \\
\mathrm{mL})\end{array}$ & $\begin{array}{l}\mathrm{AUC}_{(0-\mathrm{t})}(\mathrm{ng} \cdot \mathrm{h} / \\
\mathrm{mL})\end{array}$ & $\begin{array}{l}\mathrm{CL} / F(\mathrm{~L} / \\
\mathrm{h})\end{array}$ & $\begin{array}{l}C_{\max }(\mathrm{ng} / \\
\mathrm{mL})\end{array}$ & $t_{1 / 2}(\mathrm{~h})$ & $T_{\max }(\mathrm{h})$ & $V_{\mathrm{z}} / F(\mathrm{~L})$ \\
\hline 5 & 6 & 6 & $188.13(36)$ & $157.13(31)$ & $29.62(35)$ & $1.87(5)$ & $69.44(30)$ & $11(7-12)$ & 2738 (15) \\
\hline 10 & 182 & 228 & 273.42 (39) & $254.72(36)$ & $40.52(40)$ & $4.60(29)$ & 60.62 (69) & $8(3-36)$ & 2773 (27) \\
\hline 20 & 127 & 165 & $645.51(41)$ & $561.20(37)$ & $41.47(60)$ & $8.11(27)$ & $64.28(35)$ & $10(4-24)$ & $3288(35)$ \\
\hline
\end{tabular}

Mean and \% coefficient of variation are presented for all parameters, except $T_{\max }$, for which median (minimum-maximum) is presented

$A U C_{(0-i n f)}$ area under the curve from time 0 to infinity, $A U C_{(0-t)}$ area under the curve from time 0 to time of last quantifiable concentration, $C L / F$ total clearance, $C_{\max }$ maximum observed plasma concentration, $O b s$ observations, $t_{1 / 2}$ half-life, $T_{\max }$ time to reach maximum observed plasma concentration, $V_{z} / F$ apparent volume of distribution during the terminal phase after extravascular administration

${ }^{\mathrm{a}}$ Data were pooled from 14 clinical pharmacology studies in healthy subjects 
Table 2 Pooled non-compartmental pharmacokinetic parameters of vortioxetine following multiple doses ${ }^{\mathrm{a}}$

\begin{tabular}{lcclllllll}
\hline Dose $(\mathrm{mg})$ & Subjects $(n)$ & $\mathrm{Obs}(n)$ & $\mathrm{AI}^{\mathrm{b}}$ & $\mathrm{AUC}_{(0-24)}(\mathrm{ng} \cdot \mathrm{h} / \mathrm{mL})$ & $\mathrm{CL} / F(\mathrm{~L} / \mathrm{h})$ & $C_{\max }(\mathrm{ng} / \mathrm{mL})$ & $t_{1 / 2}(\mathrm{~h})$ & $T_{\max }(\mathrm{h})$ & $V_{\mathrm{z}} / F(\mathrm{~L})$ \\
\hline 5 & 30 & 30 & $5.17(27)$ & $175.15(45)$ & $32.96(33)$ & $8.69(42)$ & $60.05(40)$ & $7(1-12)$ & $2497(21)$ \\
10 & 242 & 242 & $4.87(34)$ & $344.00(47)$ & $38.27(60)$ & $17.92(44)$ & $58.84(45)$ & $8(0-24)$ & $3293(50)$ \\
20 & 56 & 56 & $5.68(38)$ & $645.78(39)$ & $40.11(47)$ & $33.03(38)$ & $64.23(31)$ & $8(3-14)$ & $3372(31)$ \\
\hline
\end{tabular}

Mean and \% coefficient of variation are presented for all parameters, except $T_{\max }$, for which median (minimum-maximum) is presented

$A I$ accumulation index, $A U C_{(0-24)}$ area under the plasma concentration-time curve from 0 to $24 \mathrm{~h}$ post-dose, $A U C_{(0-t a u)}$, area under the plasma concentration-time curve from 0 to the end of the dosing period, $C L / F$ total clearance, $C_{\max }$ maximum observed plasma concentration, $O b s$ observations, $t_{1 / 2}$ half-life, $T_{\max }$ time to reach maximum observed plasma concentration, $V_{z} / F$ apparent volume of distribution during the terminal phase after extravascular administration

${ }^{\mathrm{a}}$ Data were pooled from 12 clinical pharmacology studies in healthy subjects

${ }^{\mathrm{b}} \mathrm{AI}=\mathrm{AUC}_{(0-\mathrm{tau})}$ at steady state/AUC $\mathrm{AO-24)}_{(0)}$ day 1

marginally increased, suggesting that Pgp efflux function only slightly affects absorption of vortioxetine [26].

Co-administration of medication and food can reduce, delay, increase, accelerate, or have no effect on drug absorption [27]. Food (a high-fat meal) had no effect on the PK parameters of vortioxetine. The $90 \%$ confidence intervals (CIs) for $\mathrm{AUC}_{(0-t)}$ or area under the curve from time 0 to infinity $\left[\mathrm{AUC}_{(0-\text { inf })}\right]$ and $C_{\max }$ ratios (fed to fasting conditions) were within the bioequivalence range of $80-125 \%$, and no differences in time to reach maximum observed plasma concentration values were observed $[28,29]$.

\subsection{Distribution}

Data from phase I studies were pooled to estimate the apparent volume of distribution $\left(V_{\mathrm{z}} / F\right)$ of vortioxetine. Mean $V_{\mathrm{z}} / F$ following single or multiple doses of 5,10 , or $20 \mathrm{mg}$ ranged from 2500 to $3400 \mathrm{~L}$, suggesting an extensive extravascular distribution of vortioxetine (Tables 1,2). The phase I population-PK analysis showed that $V_{\mathrm{z}} / F$ for central and peripheral compartments combined was approximately 2600 L [23]. After a single oral dose of $50 \mathrm{mg}{ }^{14} \mathrm{C}$-labeled vortioxetine, drug concentration in the plasma was double the amount found in whole blood [30]. Further, radioactivity was generally not detected in red blood cells, suggesting that red blood cell distribution was negligible (data on file).

Plasma protein binding can directly affect the amount of free drug available for therapeutic effect, clearance, and toxicity [31]. Approximately $99 \%$ of vortioxetine was bound to plasma protein, with only $1 \%$ of unbound drug across all groups including healthy volunteers and individuals with various degrees of hepatic or renal impairment [32].

\subsection{Metabolism}

Vortioxetine is metabolized extensively through oxidation via cytochrome P450 (CYP) enzymes and subsequent glucuronic conjugation via uridine diphosphate glucuronosyltransferase (Fig. 1) [33, 34]. Cytochrome P450 enzymes that break down the parent compound include CYP2D6, CYP3A4/5, CYP2A6, CYP2C9, and CYP2C19, with CYP2D6 being the primary enzyme [33].

Six metabolites of vortioxetine have been observed in the plasma, including Lu AA34443 and its glucuronide, $\mathrm{Lu}$ AA39835 and its glucuronide, and two Lu AE22404 intermediate glucuronides (Fig. 1) [30]. Metabolic ratios, calculated as the ratio of the metabolite area under the curve (AUC) to the AUC of vortioxetine, were determined for each metabolite. Lu AA34443 (metabolic ratio 1) is the major metabolite of vortioxetine and constitutes 14-28\% of metabolite composition in the plasma over $72 \mathrm{~h}$ following a single dose [30]. This major metabolite is pharmacologically inactive as it does not bind to the main 5-HT receptors related to effectiveness [21]. Lu AA39835 is a minor metabolite (metabolic ratio $\leq 0.04$ ) that inhibits SERT; however, based on nonclinical data, Lu AA39835 does not penetrate the blood-brain barrier (data on file). Therefore, Lu AA39835 is thought to have minimal impact on the pharmacologic activity of vortioxetine. Given that the parent compound is thought to be responsible for invivo pharmacologic activity, it is the primary focus of this review.

\subsection{Elimination}

The estimated total clearance (CL/F) after oral administration of vortioxetine was found to range from 30 to 41 $\mathrm{L} / \mathrm{h}$ and the half-life $\left(t_{1 / 2}\right)$ from 59 to $69 \mathrm{~h}$ after single or multiple doses (Tables 1,2). Results from the phase I population-PK analysis were similar with $33 \mathrm{~L} / \mathrm{h}$ and $66 \mathrm{~h}$ for mean oral clearance and mean $t_{1 / 2}$, respectively [23].

Vortioxetine is almost entirely eliminated by the liver; renal clearance of the parent drug contributed to $<1 \%$ of $\mathrm{CL} / F$, while hepatic clearance accounted for the other $99 \%$ [21]. In an open-label study, approximately $85 \%$ of drugrelated material was recovered in the urine $(59 \%)$ and feces (26\%) within $360 \mathrm{~h}$ following a single dose of 50-mg 


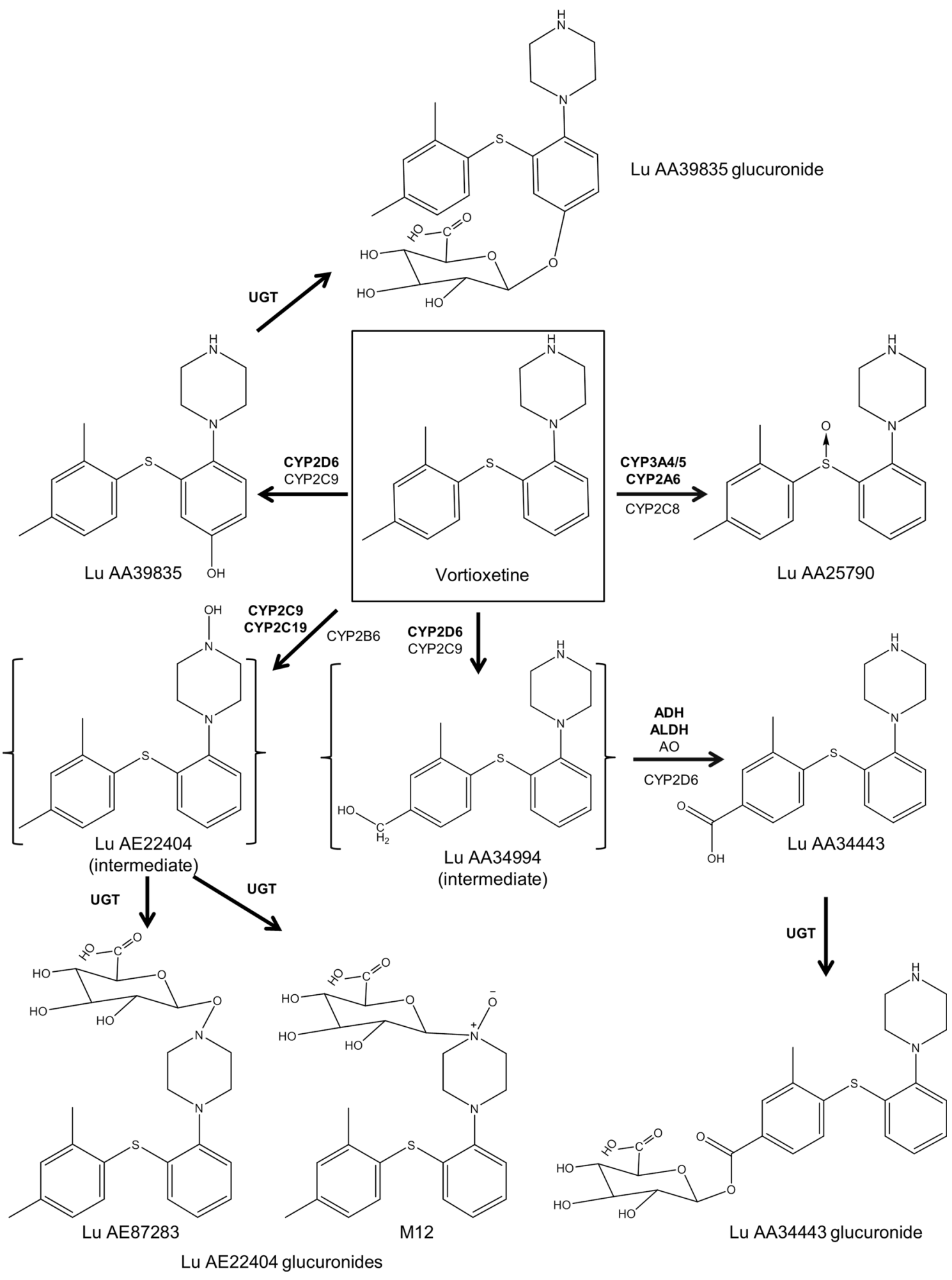

Fig. 1 Enzymatic degradation of vortioxetine. Cytochrome P450 (CYP) 2D6, CYP3A4/5, CYP2A6, CYP2C9, CYP2C19, alcohol dehydrogenase (ADH), aldehyde dehydrogenase (ALDH), and uridine diphosphate glucuronosyltransferase (UGT) are considered to be the

primary enzymes involved in the metabolism of vortioxetine. The intermediates were detected in vitro, but not in vivo. $A O$ aldehyde oxidase 
radiolabeled $\left({ }^{14} \mathrm{C}\right)$ vortioxetine [30]. Most of the detectable drug-related material was secreted in urine and feces as inactive metabolites, with negligible amounts of parent drug $(1.5 \%)$ detected in the feces only.

\subsection{Dose Proportionality and Time Dependency}

Dose proportionality characterizes the relationship between drug dosage and its PK effects, and can be clinically important for predicting the effects of dose adjustments [35]. Dose proportionality of single and multiple doses of vortioxetine was evaluated using pooled data from phase I studies with single doses of $2.5-75 \mathrm{mg}$ or multiple daily doses of $2.5-60 \mathrm{mg}$ and indicated dose proportionality within the studied dose ranges [21]. The linear relationships between dose and exposure $\left(C_{\max }\right.$ and AUC) are further illustrated in Tables 1 and 2.

Based on the pooled non-compartmental analysis, the steady-state $\mathrm{CL} / F$ values following multiple doses were similar to the CL/F values following single doses (Tables 1,2). Overall, no apparent time dependency or non-linearity was observed in the population-PK model. Estimations based on the distribution of $t_{1 / 2}$ showed that approximately $90 \%$ of individuals reached $90 \%$ of steadystate exposure after 12 days of multiple-dose administration of vortioxetine [23]. The accumulation index for vortioxetine, defined as the mean area under the curve from time 0 to $24 \mathrm{~h}\left[\mathrm{AUC}_{(0-24)}\right]$ ratio between the steady-state condition and a single dose, was 5-6 following multiple doses of 5-20 mg (Table 2), consistent with its long $t_{1 / 2}$ $(59-69 \mathrm{~h})$ (Tables 1, 2). No time dependency has been observed for the other PK parameters of vortioxetine.

\section{Special Populations}

The impact of intrinsic factors including sex, age, race/ ethnicity, body size, hepatic and renal status, and metabolic status on vortioxetine pharmacokinetics was evaluated in dedicated phase I studies and/or in the phase I populationPK analysis across 26 studies [23]. Except for the inferred CYP2D6 metabolic phenotypes, none of the intrinsic factors studied affected exposure to vortioxetine. Thus, vortioxetine can be given to diverse patients without dose adjustment, but dose may be adjusted based on individual patient response, if needed [1].

\subsection{Sex}

A single-dose study compared 24 healthy male subjects with 24 healthy female subjects and found that $\mathrm{AUC}_{(0-\mathrm{t})}$ and $C_{\max }$ values were 18 and $16 \%$ higher in female subjects than in male subjects, respectively, although these increases were not considered clinically meaningful [36]. Similar results were observed following multiple doses, with $\mathrm{AUC}_{(0-24)}$ and $C_{\max }$ values 27 and $24 \%$ higher, respectively, in female subjects than in male subjects (Fig. 2).

The phase I population-PK analysis found that sex was not a significant covariate in the final model after correction for height, one of the most significant covariates for body size [23]. Overall, sex differences on vortioxetine exposure were not considered clinically significant, especially after accounting for weight or height differences.

\subsection{Age}

The effects of age on the pharmacokinetics of vortioxetine were evaluated in a phase I study of 24 young volunteers aged 18-45 years and 24 elderly volunteers aged 65-85 years [36]. Following single doses of vortioxetine $10 \mathrm{mg}$, a slightly higher $\mathrm{AUC}_{(0-\mathrm{t})}(4 \%)$ and lower $C_{\max }$ (11\%) were observed in the elderly than the young subjects [36]. Furthermore, $\mathrm{AUC}_{(0-24)}$ and $C_{\max }$ were 27 and $23 \%$ higher, respectively, in the elderly than the young subjects following multiple 10-mg doses (Fig. 2).

Using the phase I population-PK model, simulations of vortioxetine $(20 \mathrm{mg})$ pharmacokinetics at steady state were conducted for an average 18-year-old, 40-year-old, and 75-year-old person to determine the effect of age [23]. Compared with a 40-year-old individual, the 75-year-old individual had $C_{\max }$ and $\mathrm{AUC}_{(0-24)}$ values 38 and $40 \%$ higher, respectively, while the 18-year-old individual had $C_{\max }$ and $\mathrm{AUC}_{(0-24)}$ values 14 and $15 \%$ lower, respectively. These estimated modest increases or decreases in vortioxetine exposure were not considered clinically meaningful [23]. Together, these data do not support the need for dose adjustments in the elderly population. In fact, a recent meta-analysis of 12 short-term MDD studies indicated that patients aged 55 years and older could be initiated on vortioxetine doses in line with the overall adult population; however, doses may be lowered to $5 \mathrm{mg}$ daily over tolerability concerns [37].

\subsection{Race/Ethnicity}

In one study, 24 healthy white and 24 healthy black volunteers were administered a single dose of vortioxetine $10 \mathrm{mg}$ followed by daily doses for 14 days [36], while a second study evaluated multiple doses of $2.5,5$, and $20 \mathrm{mg}$ in 18 white individuals and 18 Japanese male individuals. Overall, compared with values obtained in the white volunteers, $C_{\max }$ and $\mathrm{AUC}_{(0-24)}$ values in the black participants were 33 and 25\% higher, respectively (Fig. 2), and values in the Japanese study participants were 9 and $8 \%$ higher, respectively (data on file). The findings from the two studies were corroborated by the phase I population- 
Fig. 2 Impact of intrinsic factors on vortioxetine pharmacokinetics following multiple doses. Forest plot illustrating the fold change in area under the curve (AUC) and maximal observed plasma concentration $\left(C_{\max }\right)$ between test and reference populations. CI confidence interval, ESRD early-stage renal disease

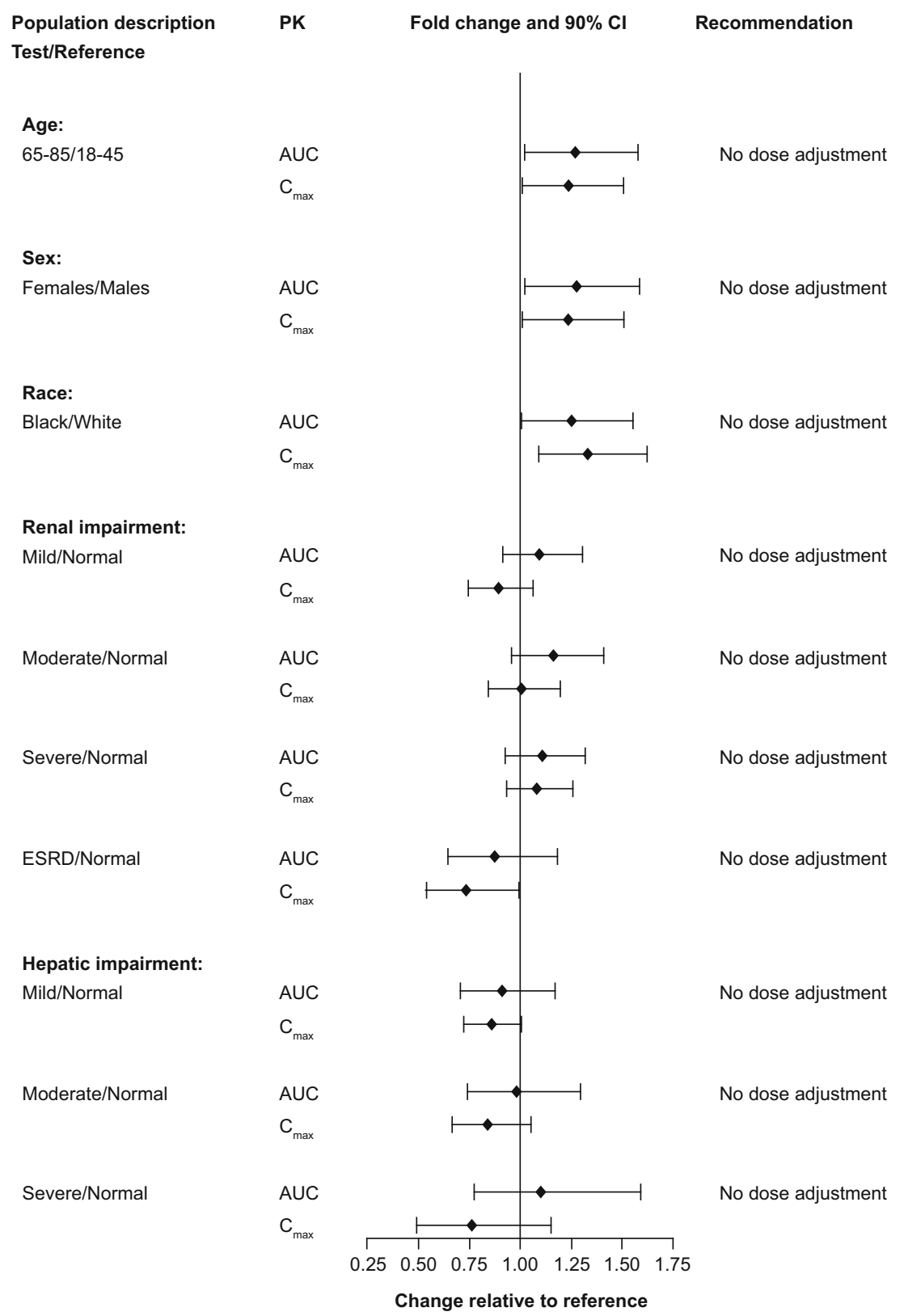

population-PK model for vortioxetine $(20 \mathrm{mg})$ revealed that, compared with an average person $175 \mathrm{~cm}$ tall, a shorter person $(155 \mathrm{~cm})$ had $5 \%$ higher $C_{\max }$ and $\mathrm{AUC}_{(0-24)}$ values and a taller person $(187 \mathrm{~cm})$ had $4 \%$ lower values; however, given the small differences, the variability was not considered clinically meaningful [23].

\subsection{Hepatic Impairment}

The effect of hepatic impairment on vortioxetine pharmacokinetics was studied in individuals with mild $(n=8)$, moderate $(n=8)$, and severe $(n=6)$ impairment (ChildPugh classification) and in the same number of healthy, race-, age-, and sex-matched individuals. All study participants 
received a single dose of open-label vortioxetine $10 \mathrm{mg}$ (except those with severe impairment and their matched healthy controls, where vortioxetine $5 \mathrm{mg}$ was used) [38].

There were no differences in the fraction of unbound parent drug when comparing hepatically impaired individuals with healthy participants; therefore, only total exposure was compared between the two groups. $\mathrm{AUC}_{(0-\mathrm{t})}$ was up to $9 \%$ lower and $C_{\max }$ up to $16 \%$ lower in those with mild-to-moderate hepatic impairment than in the healthy cohorts (Fig. 2). Among individuals with severe hepatic impairment, $\mathrm{AUC}_{(0-\mathrm{t})}$ was $10 \%$ higher and $C_{\max }$ $24 \%$ lower than in healthy controls [38]. Plasma exposure to the major metabolite Lu AA34443 was lower in individuals with moderate and severe hepatic impairment than in healthy controls. Exposure to the minor metabolite Lu AA39835 was lower in individuals with moderate impairment [38]. However, given that the major metabolite is pharmacologically inactive and that the minor metabolite has a low metabolic ratio $(\leq 0.04)$, the reported changes in exposure are likely to have minimal impact on the pharmacologic effect of vortioxetine. The relationship between exposure to vortioxetine or its metabolites and Child-Pugh scores was non-significant, even though the drug was heavily metabolized by the liver [38]. Overall, changes in vortioxetine exposure in individuals with hepatic impairment were not considered clinically meaningful, and dose adjustments are not necessary in patients with mild, moderate, or severe hepatic impairment [38].

\subsection{Renal Impairment}

To assess the effects of renal impairment on vortioxetine, individuals with mild $(n=8)$, moderate $(n=8)$, severe $(n=9)$, or end-stage renal disease $(n=8)$ and an equal number of healthy controls for each subgroup received a single dose of open-label vortioxetine $10 \mathrm{mg}$ [32]. Creatinine clearance, a measure of renal function, ranged from $<30 \mathrm{~mL} / \mathrm{min}$ in those with severe renal impairment to $>80 \mathrm{~mL} / \mathrm{min}$ in those with normal renal function. Further, there was no difference in vortioxetine plasma protein binding between renally compromised patients and healthy controls [32]. The $\mathrm{AUC}_{(0-\mathrm{t})}$ values were 9, 16, and $11 \%$ higher among individuals with mild, moderate, or severe renal impairment, respectively, than in the healthy controls, and $C_{\max }$ values were within $11 \%$ of healthy controls. In contrast, participants with end-stage renal disease had $\mathrm{AUC}_{(0-\mathrm{t})}$ and $C_{\max }$ values that were 13 and $27 \%$ lower, respectively, than those for the healthy controls (Fig. 2) [32]. However, the magnitude of these changes was not considered clinically meaningful. Using a linear regression model, a statistically significant relationship was observed between $\mathrm{AUC}_{(0-\mathrm{t})}$ and creatinine clearance, but not between $C_{\max }$ and creatinine clearance. The predicted vortioxetine exposure was within $30 \%$ of that expected in the healthy control group [32]. These results suggest that clinical dose adjustments are not considered necessary in patients with various degrees of renal impairment.

\subsection{Metabolic Status (CYP2D6 and CYP2C19 Inferred Phenotypes)}

Vortioxetine is primarily metabolized by the CYP2D6 enzyme, and less so by CYP2C19 [33]. Genetic variations in the CYP2D6 gene can affect drug exposure, depending on whether a patient is an intermediate metabolizer (IM), extensive metabolizer (EM), poor metabolizer (PM), or ultrarapid metabolizer (UM) of the drug [39]. Among the 887 healthy volunteers included in the phase I population-PK analysis, there were 540 CYP2D6 EMs (61\%) and 38 CYP2D6 PMs (4\%) [23]. Oral clearance of vortioxetine was approximately two-fold higher in the EMs than in the PMs. Based on CYP2D6 status, estimated CL/F (L/h) was 52.9, 34.1, 26.6, and 18.1 for UMs, EMs, IMs, and PMs, respectively [23]. Although the population mean AUC for CYP2D6 UMs is expected to be $35.5 \%$ lower than that in the CYP2D6 EMs, there was a significant AUC overlap between CYP2D6 $\mathrm{UMs}$ and EMs owing to inter-subject variability. Dose adjustment for CYP2D6 UMs is not necessary.

Exposure to vortioxetine in CYP2D6 PMs is expected to be twice as high as in CYP2D6 EMs. Based on tolerability analyses using phase I data grouped by CYP2D6-predicted phenotypes, no clinically relevant differences were observed for the overall incidence of adverse events (e.g., nausea, diarrhea, headache) between CYP2D6 PMs and non-PMs, possibly because of the longer $t_{1 / 2}$ and the gradual accumulation of the plasma concentration of vortioxetine over a much longer period of time in CYP2D6 PMs. Therefore, a routine CYP2D6 genotyping test before starting vortioxetine treatment is not required. Depending on individual patient response, a dose adjustment may be considered, with a maximum recommended dose of $10 \mathrm{mg}$ for known CYP2D6 PMs.

Phase I population-PK analysis also explored variations in the CYP2C19 gene and identified 548 CYP2C19 EMs (62\%) and 44 CYP2C19 PMs (5\%). Extensive metabolizers had on average 1.4 times the oral clearance of PMs [23]. However, this higher vortioxetine exposure was not considered clinically meaningful; therefore, dose adjustment is not necessary based on CYP2C19 status.

\section{Drug Interaction Evaluations}

Besides CYP2D6, several other CYP enzymes are involved in vortioxetine metabolism (Fig. 1). Consequently, other drugs that are substrates of the same CYP enzymes can 
affect vortioxetine pharmacokinetics or, in turn, have their pharmacokinetics affected by vortioxetine and/or its metabolites. To observe the effects of potential drug-drug interactions, 11 studies have been conducted in healthy volunteers.

\subsection{Impact of Other Drugs on Vortioxetine}

Several drug-drug interaction studies have evaluated the effect of other drugs including bupropion, rifampin, ketoconazole, fluconazole, omeprazole, ethanol, and aspirin on vortioxetine pharmacokinetics (Fig. 3). Bupropion is a CYP2B6 substrate, and its active metabolites threohydrobupropion and erythrohydrobupropion are strong CYP2D6 inhibitors. When bupropion $150 \mathrm{mg}$ was co-administered twice daily with vortioxetine $10 \mathrm{mg}$ once daily in 44 participants, vortioxetine exposure approximately doubled with $\mathrm{AUC}_{(0-24)}$ increasing 2.3-fold and $C_{\max }$ increasing 2.1-fold [26] (Fig. 3). Adverse events, including nausea, headache, vomiting, and insomnia, were three times as likely when bupropion was added to vortioxetine monotherapy as when vortioxetine was added to bupropion therapy or when vortioxetine was administered alone. Based on these results, it is recommended to reduce the vortioxetine dose by half when co-administered with bupropion or other strong inhibitors of CYP2D6 (e.g., fluoxetine, paroxetine, or quinidine).

Rifampin is a broad CYP inducer. When rifampin was given at a $600-\mathrm{mg}$ dose daily to 14 male and female individuals along with vortioxetine $20 \mathrm{mg}$ in an interaction study, it was found that vortioxetine $\mathrm{AUC}_{(0-\mathrm{t})}$ decreased by $72 \%$ and $C_{\max }$ decreased by $51 \%$ (Fig. 3). The combination was well tolerated [26]. Vortioxetine is extensively metabolized via multiple CYP isozymes. Although CYP2D6, the primary enzyme involved in the metabolism of vortioxetine, is not subject to CYP induction by rifampin, the metabolic enzyme activities of other isozymes, such as CYP3A4/5, CYP2C19, CYP2C9, CYP2C8, and CYP2B6, may be induced by treatment with rifampin and lead to increased oral clearance and decreased exposure. It is recommended to consider increasing the vortioxetine dose when rifampin or other strong CYP inducers (e.g., carbamazepine or phenytoin) are co-administered for more than 14 days. The maximum recommended dose should not exceed three times the original vortioxetine dose.

Another interaction study in 33 male and female individuals found that once-daily co-administration of ketoconazole $400 \mathrm{mg}$ (a CYP3A4/5 and Pgp inhibitor) or fluconazole $200 \mathrm{mg}$ (a CYP2C9, CYP2C19, and CYP3A4/ 5 inhibitor) with vortioxetine $10 \mathrm{mg}$ increased the $\mathrm{AUC}_{(0-\mathrm{t})}$ of vortioxetine by 30 and $46 \%$, respectively, and $C_{\max }$ by 26 and 15\%, respectively [26]. Given the modest increases, these findings were not considered clinically meaningful.
Interactions between vortioxetine and ethanol, aspirin, or omeprazole (a CYP2C19 inhibitor and substrate) were assessed in three independent studies [26, 40, 41]. In all cases, co-administration of ethanol, aspirin, or omeprazole had no effect on exposure to vortioxetine (Fig. 3).

\subsection{Impact of Vortioxetine on Other Drugs}

Vortioxetine and its metabolites showed no inhibitory invitro effect on CYP1A2, CYP2A6, CYP2E1, CYP3A4/5, or CYP2D6 enzymes. The parent drug and certain metabolites, however, had an inhibitory effect on the CYP2C19, CYP2C9, and CYP2C8 enzymes as demonstrated in inhibition studies performed in vitro using pooled human liver microsomes and CYP isozyme-specific probe substrates (data on file). Nevertheless, given that the ratios of maximum plasma concentration for the inhibitor [vortioxetine or its metabolites] (I) and dissociation constant of the inhibitor $(\mathrm{Ki})$ were much smaller than 0.1 , the inhibition was not considered clinically relevant. Vortioxetine was found to be a poor substrate and a weak inhibitor of Pgp transport in vitro [25, 26, 42]. Therefore, the potential for vortioxetine to inhibit Pgp efflux is considered low (I/ half maximal inhibitory concentration $<0.1$ ) [42].

No effect was seen on the pharmacokinetics of warfarin, diazepam, caffeine, tolbutamide, ethinyl estradiol, levonorgestrel, midazolam, aspirin, ethanol, or lithium when given along with vortioxetine (Fig. 4). However, vortioxetine was noted to affect the pharmacokinetics of certain compounds or their metabolites as discussed below.

The effect of multiple doses of vortioxetine on bupropion (CYP2B6 substrate) and omeprazole (CYP2C19 substrate) was evaluated [26]. The 90\% CIs for area under the curve from time 0 to $12 \mathrm{~h}$ (bupropion) and $\mathrm{AUC}_{(0-\mathrm{inf})}$ (omeprazole) were within the $80-125 \%$ limits. For $C_{\max }$, the upper limit of the $90 \%$ CI was only slightly exceeded for bupropion (129\%) and omeprazole (130\%) (Fig. 4). Overall, these results indicate that multiple doses of vortioxetine had no apparent effect on the pharmacokinetics of bupropion or omeprazole and that vortioxetine is not an inhibitor or inducer of CYP2B6 or CYP2C19 [26].

To observe the effects on the pharmacokinetics of substrates of different CYP enzymes, 24 male and female individuals were given a cocktail of caffeine (CYP1A2 substrate), tolbutamide (CYP2C9 substrate), dextromethorphan (CYP2D6 substrate), and midazolam (CYP3A4/5 substrate) along with vortioxetine [43]. Notably, the $90 \% \mathrm{CI}$ for $\mathrm{AUC}_{(0-\mathrm{inf})}$ and $C_{\max }$ of all compounds were within the limits of $80-125 \%$, with one exception. For dextromethorphan, the $90 \%$ CI lower limit was below $80 \%$ with values for $\mathrm{AUC}_{(0-\text { inf })}$ at $58 \%$ and $C_{\max }$ at $68 \%$ (Fig. 4). Overall, these data indicate that multiple doses of vortioxetine have no substantial effect on the 
Fig. 3 Impact of other drugs on vortioxetine pharmacokinetics. Forest plot illustrating fold change in the area under the curve (AUC) or maximal $\left(C_{\max }\right)$ of vortioxetine when coadministered with other drugs relative to vortioxetine alone. Dashed lines indicate no effect boundaries [ $90 \%$ confidence interval (CI) 80-125]. Pgp

P-glycoprotein observed plasma concentration

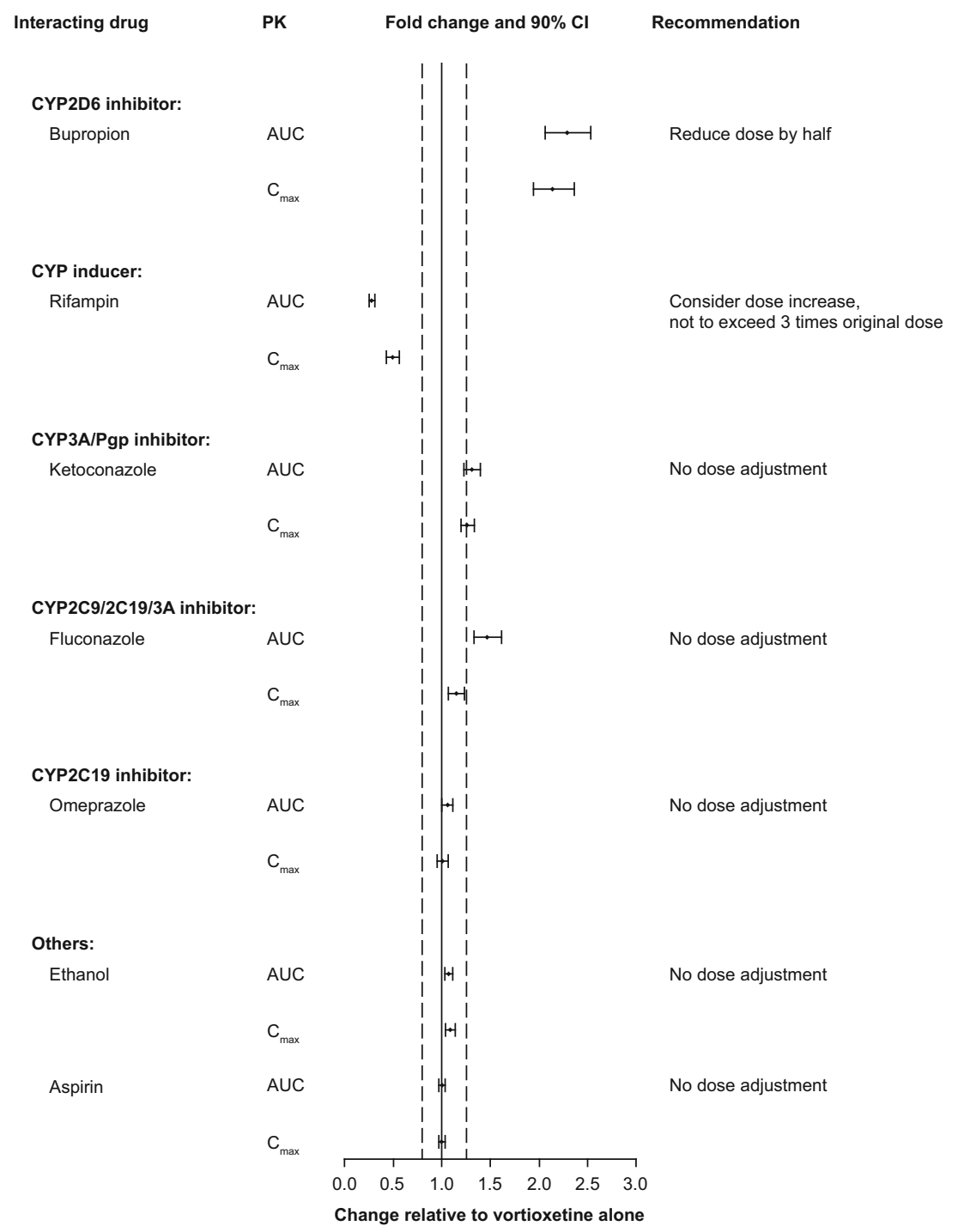

pharmacokinetics of any of the cocktail components, implying that vortioxetine is not an inducer or inhibitor of CYP1A2, CYP2C9, CYP2D6, and CYP3A4/5 substrates [43].

\section{Clinical Pharmacodynamics}

Phase I clinical PD studies on vortioxetine investigated parameters such as drug occupancy at SERT, drug effects on neurotransmitters in the cerebrospinal fluid, cardiac repolarization, driving performance, sleep architecture, and cognition. In addition, the impact of vortioxetine on the pharmacodynamics of other drugs was also explored in several drug-drug interaction studies.

The occupancy of vortioxetine at SERT in different brain regions was studied in two positron emission tomography studies using ${ }^{11} \mathrm{C}-\mathrm{MADAM},{ }^{11} \mathrm{C}-\mathrm{WAY}$ -
100635, or ${ }^{11} \mathrm{C}$-DASB ligands $[44,45]$. In the first study, 15 young male individuals received vortioxetine $(2.5,10$, or $60 \mathrm{mg}$ once daily) for 9 consecutive days; SERT occupancy was measured in regions of interests including the frontal cortex, insular, hippocampus, and raphe nuclei. The highest SERT occupancy was detected in the raphe nuclei from 15 young male individuals, with median occupancy values of $25,53 \%$, and $98 \%$ at day 9 for the 2.5-, 10-, and 60-mg doses, respectively [45]. Similar results were obtained in 35 Japanese and white male individuals, with occupancies of approximately 50 and $65 \%$ for the 5- and $10-\mathrm{mg}$ therapeutic doses, respectively, and above $80 \%$ for the 20-mg dose [44]. These results indicate that vortioxetine may be efficacious at SERT occupancy as low as $50 \%$, given that occupancy is not saturated at therapeutic doses. In contrast, occupancy of $80 \%$ is required for selective serotonin reuptake inhibitors and serotonin norepinephrine reuptake inhibitors to achieve therapeutic 
Fig. 4 Impact of vortioxetine on the pharmacokinetics of other drugs. Forest plot illustrating fold change in drug's area under the curve (AUC) or maximal observed plasma concentration $\left(C_{\max }\right)$ when co-administered with vortioxetine relative to drug alone. Dashed lines indicate no effect boundaries [90\% confidence interval (CI) 80-125]

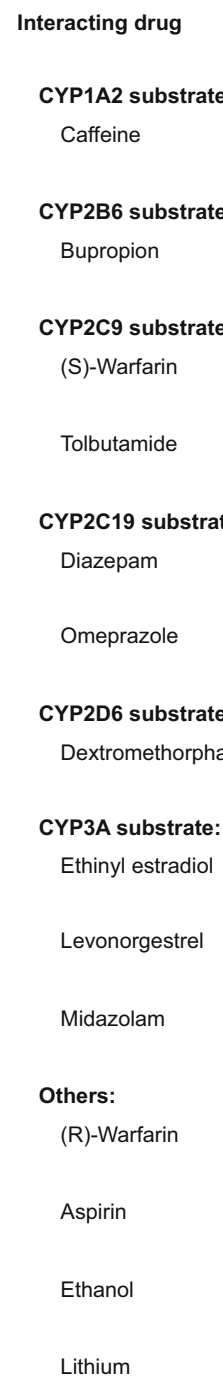

effects [46]. The results also suggest that vortioxetine may mediate its antidepressant activity through actions on 5-HT receptors in addition to SERT inhibition [44].

The effect of vortioxetine on neurotransmitters in the cerebrospinal fluid was investigated in a study of 15 healthy male participants following single and multiple doses of vortioxetine $20 \mathrm{mg}$ over 14 days (data on file). Consistent effects were observed for 5-HT and its metabolite 5-hydroxyindoleacetic acid. Overall, PD parameters (area under the effect-time curve from 0 to $24 \mathrm{~h}$ and maximal analyte concentration from 0 to $24 \mathrm{~h}$ ) increased from baseline for 5-HT following single and multiple doses of vortioxetine $20 \mathrm{mg}$ and decreased for 5-hydroxyindoleacetic acid after multiple doses only. These results indicate a measurable effect of vortioxetine
PK

Fold change and $90 \% \mathrm{Cl}$

Recommendation

No dose adjustment

No dose adjustment

No dose adjustment

No dose adjustment

No dose adjustment

No dose adjustment

No dose adjustment

No dose adjustment

No dose adjustment

No dose adjustment

No dose adjustment

No dose adjustment

No dose adjustment

No dose adjustment

on cerebrospinal fluid levels of 5-HT and 5-hydroxyindoleacetic acid.

The effects of vortioxetine on cognitive function were investigated by assessing functional connectivity in two resting state networks, the default mode network (DMN) and salience network. Using functional magnetic resonance imaging, vortioxetine was found to reverse DMN abnormalities reported in depressed patients. In addition, vortioxetine modulated dorsal DMN and anterior salience network expressions in an overlapping region of the medial frontal gyrus, which has been implicated as a key nodal point of salience network-DMN interaction [47]. These data suggest that the effect of vortioxetine on resting-state network connectivity may account for its reported benefits on cognitive function. 
A multiple-dose, parallel-group study in 328 young male individuals evaluated the effect of vortioxetine on cardiac repolarization [48]. No correlation was observed between plasma concentration of vortioxetine or its metabolites and time-matched, baseline-adjusted corrected QT intervals at either 10- or 40-mg doses. Therefore, according to the International Conference on Harmonisation E14 definitions, the administration of vortioxetine (10 or $40 \mathrm{mg}$ once daily) is unlikely to affect cardiac repolarization [48].

Furthermore, vortioxetine was found not to impair driving, cognitive function, or psychomotor skills in a study of 21 young male and female individuals who received once-daily doses of vortioxetine $10 \mathrm{mg}$ [49]. A study of 24 participants compared the effects of vortioxetine $(20 \mathrm{or} 40 \mathrm{mg}$ ) or paroxetine $(20 \mathrm{mg})$, a potent serotonin reuptake inhibitor, with placebo on sleep architecture [50]. There was a significant reduction in rapid eye movement (REM) sleep, an increase in REM onset latency, and a decrease in time spent in REM sleep for active treatments compared with placebo. Of note, the relationship between SERT occupancy and REM suppression showed different profiles for the two drugs with a direct relationship between occupancy and REM suppression observed with paroxetine, but not with vortioxetine [50]. These data suggest that vortioxetine has a clinical pharmacologic profile distinguishable from paroxetine, whereby SERT inhibition may not be the only contributor to REM suppression. Overall, these results are likely related to multimodal interactions of vortioxetine with 5-HT receptors.

Multiple drug-drug interaction studies showed that vortioxetine largely did not affect the pharmacodynamics of other central nervous system active agents such as diazepam and alcohol [40]. More specifically, vortioxetine did not increase the mental and motor impairment effects of alcohol when measured by computerized neuropsychologic tests for various cognitive domains. In the diazepam interaction study, co-administration of vortioxetine did not have a statistically significant effect on neuropsychologic tests used to assess cognitive domains [40].

The use of drugs that interfere with serotonin reuptake inhibition, including vortioxetine, may increase the risk of bleeding events. The impact of vortioxetine on two drugs that affect hemostasis was examined in healthy subjects. Results from a 14-day drug-drug interaction study with a $10-\mathrm{mg}$ dose of vortioxetine and stable warfarin doses $(1-10 \mathrm{mg})$ indicated that there was no difference in the International Normalized Ratio or prothrombin times between the groups on day 14. Similarly, vortioxetine at the 10-mg dose when added to the 150-mg dose of aspirin did not change arachidonic acid-, adenosine diphosphate-, or collagen-induced platelet aggregation [41]. In an oral contraceptive study of 25 young female individuals, sex hormones including luteinizing hormone, follicle-stimulating hormone, sex hormone-binding globulin, estradiol, and progesterone were also unaffected by co-administration of vortioxetine and ethinyl estradiol combined with levonorgestrel [26, 51].

Finally, as with other serotonergic antidepressant therapy, treatment with vortioxetine may lead to the development of serotonin syndrome, especially when used concomitantly with other serotonergic drugs (including triptans, tricyclic antidepressants, fentanyl, lithium, tramadol, tryptophan, buspirone, and St. John's Wort) and with drugs that impair the metabolism of serotonin (in particular monoamine oxidase inhibitors) [52].

\section{Summary}

Vortioxetine is a novel antidepressant with favorable PK/ PD properties characterized by a large volume of distribution, a medium clearance, and a relatively long terminal $t_{1 / 2}$. Vortioxetine has an oral bioavailability of $75 \%$ and is primarily metabolized by the liver through the CYP2D6 enzyme pathway, with the parent drug responsible for the therapeutic effect. Because vortioxetine is primarily metabolized by CYP2D6, PMs of CYP2D6 exhibit exposure to vortioxetine twice as high as CYP2D6 EMs.

Intrinsic factors such as age, sex, race/ethnicity, body size, and hepatic and renal impairment had no clinically meaningful effects on vortioxetine exposure. Food intake or alcohol consumption did not affect exposure to vortioxetine. It has been reported that selected antidepressants, including selective serotonin reuptake inhibitors and serotonin norepinephrine reuptake inhibitors, have the potential to impact the pharmacokinetics and/or pharmacodynamics of other central nervous system agents or medications. The drug interaction potentials with vortioxetine were extensively evaluated in 11 dedicated clinical studies based on the mechanism of drug metabolism and anticipated clinical use. According to available clinical data, vortioxetine has minimal effect on the PK parameters of co-administered drugs such as bupropion, warfarin, diazepam, omeprazole, aspirin, lithium, ethanol, and oral contraceptives. In addition, vortioxetine did not have significant effects on PD parameters, such as the International Normalized Ratio, platelet aggregation, sex hormone levels, cognitive function, motor skills, or neuropsychologic tests. Administration of vortioxetine has no effect on cardiac repolarization. These studies have shown that vortioxetine did not have any clinically meaningful effects on the pharmacokinetics and/or pharmacodynamics of the concomitant agents or medications mentioned above. Exposure to vortioxetine was increased when given with a strong CYP2D6 inhibitor bupropion and decreased when given with a broad CYP 
inducer rifampin. Concomitant therapy was generally well tolerated, except with bupropion; addition of bupropion to vortioxetine monotherapy increased the incidence of nausea, vomiting, and insomnia [26]. Most adverse events in drug-drug interaction studies were mild or moderate [26]. Vortioxetine was also well tolerated regardless of the degree of renal or hepatic impairment $[32,38]$.

In this review, we summarized vortioxetine PK/PD data from individual phase I studies and phase I population-PK analyses performed in healthy individuals. The PK/PD results reported in patients with MDD or generalized anxiety disorder were generally consistent with those in healthy individuals. Conforming to what was observed in healthy individuals, intrinsic factors such as age, renal impairment, hepatic impairment, and body size did not have a clinically relevant effect on vortioxetine exposure in patients with MDD or generalized anxiety disorder [53]. Overall, PK parameters of vortioxetine in patients with MDD and generalized anxiety disorder were generally similar to those in healthy individuals presented here, further highlighting the applicability of phase I results in healthy individuals to patients with the target disease.

Acknowledgements Medical writing assistance was provided by Reem Berro, PhD, Neil Patel, PharmD, and Bomina Yu, PhD, CMPP, of inVentiv Medical Communications and supported by Takeda Pharmaceuticals U.S.A., Inc., and H. Lundbeck A/S.

\section{Compliance with Ethical Standards}

Funding This study was sponsored by Takeda Development Center Americas, Inc., and H. Lundbeck A/S.

Conflict of interest Grace Chen is an employee of Takeda Development Center Americas, Inc. Astrid-Maria Højer and Johan Areberg are employees of $\mathrm{H}$. Lundbeck A/S. George Nomikos is a former employee of Takeda Development Center Americas, Inc.

Ethics approval All procedures performed in studies involving human participants were in accordance with the ethical standards of the institutional and/ or national research committee and with the 1964 Helsinki Declaration and its later amendments or comparable ethical standards.

Consent to participate Informed consent was obtained from all individual participants included in the study.

Open Access This article is distributed under the terms of the Creative Commons Attribution-NonCommercial 4.0 International License (http://creativecommons.org/licenses/by-nc/4.0/), which permits any noncommercial use, distribution, and reproduction in any medium, provided you give appropriate credit to the original author(s) and the source, provide a link to the Creative Commons license, and indicate if changes were made.

\section{References}

1. Lundbeck. Trintellix [package insert]. Deerfield (IL): Lundbeck; 2016.

2. Koenig AM, Thase ME. First-line pharmacotherapies for depression: what is the best choice? Pol Arch Med Wewn. 2009;119(7-8):478-86.

3. Lomas J, Llewellyn A, Soares M, Simmonds M, Wright K, Eastwood A, et al. The clinical and cost effectiveness of vortioxetine for the treatment of a major depressive episode in patients with failed prior antidepressant therapy: a critique of the evidence. Pharmacoeconomics. 2016;34(9):901-12. https://doi. org/10.1007/s40273-016-0417-9.

4. Connolly KR, Thase ME. Vortioxetine: a new treatment for major depressive disorder. Expert Opin Pharmacother. 2016;17(3): 421-31. https://doi.org/10.1517/14656566.2016.1133588.

5. Brignone M, Diamand F, Painchault C, Takyar S. Efficacy and tolerability of switching therapy to vortioxetine versus other antidepressants in patients with major depressive disorder. Curr Med Res Opin. 2016;32(2):351-66. https://doi.org/10.1185/ 03007995.2015.1128404.

6. Sanchez C, Asin KE, Artigas F. Vortioxetine, a novel antidepressant with multimodal activity: review of preclinical and clinical data. Pharmacol Ther. 2015;145:43-57. https://doi.org/ 10.1016/j.pharmthera.2014.07.001.

7. Garnock-Jones KP. Vortioxetine: a review of its use in major depressive disorder. CNS Drugs. 2014;28(9):855-74. https://doi. org/10.1007/s40263-014-0195-x.

8. Bang-Andersen B, Ruhland T, Jorgensen M, Smith G, Frederiksen $K$, Jensen KG, et al. Discovery of 1-[2-(2,4dimethylphenylsulfanyl)phenyl]piperazine (Lu AA21004): a novel multimodal compound for the treatment of major depressive disorder. J Med Chem. 2011;54(9):3206-21. https://doi.org/ $10.1021 / \mathrm{jm} 101459 \mathrm{~g}$.

9. Pehrson AL, Sanchez C. Serotonergic modulation of glutamate neurotransmission as a strategy for treating depression and cognitive dysfunction. CNS Spectr. 2014;19(2):121-33. https://doi. org/10.1017/S1092852913000540.

10. Mork A, Montezinho LP, Miller S, Trippodi-Murphy C, Plath N, $\mathrm{Li}$ Y, et al. Vortioxetine (Lu AA21004), a novel multimodal antidepressant, enhances memory in rats. Pharmacol Biochem Behav. 2013;105:41-50. https://doi.org/10.1016/j.pbb.2013.01. 019.

11. Pehrson AL, Cremers T, Betry C, van der Hart MG, Jorgensen L, Madsen M, et al. Lu AA21004, a novel multimodal antidepressant, produces regionally selective increases of multiple neurotransmitters: a rat microdialysis and electrophysiology study. Eur Neuropsychopharmacol. 2013;23(2):133-45. https://doi.org/10. 1016/j.euroneuro.2012.04.006.

12. Stahl SM. Modes and nodes explain the mechanism of action of vortioxetine, a multimodal agent (MMA): blocking 5HT3 receptors enhances release of serotonin, norepinephrine, and acetylcholine. CNS Spectr. 2015;20(5):455-9. https://doi.org/10. 1017/S1092852915000346.

13. Stahl SM. Modes and nodes explain the mechanism of action of vortioxetine, a multimodal agent (MMA): modifying serotonin's downstream effects on glutamate and GABA (gamma amino butyric acid) release. CNS Spectr. 2015;20(4):331-6. https://doi. org/10.1017/S1092852915000334. 
14. Stahl SM. Modes and nodes explain the mechanism of action of vortioxetine, a multimodal agent (MMA): enhancing serotonin release by combining serotonin (5HT) transporter inhibition with actions at 5HT receptors (5HT1A, 5HT1B, 5HT1D, 5HT7 receptors). CNS Spectr. 2015;20(2):93-7. https://doi.org/10.1017/ S1092852915000139.

15. Alvarez E, Perez V, Dragheim M, Loft H, Artigas F. A doubleblind, randomized, placebo-controlled, active reference study of Lu AA21004 in patients with major depressive disorder. Int $\mathbf{J}$ Neuropsychopharmacol. 2012;15(5):589-600. https://doi.org/10. 1017/S1461145711001027.

16. Baldwin DS, Loft H, Dragheim M. A randomised, double-blind, placebo controlled, duloxetine-referenced, fixed-dose study of three dosages of Lu AA21004 in acute treatment of major depressive disorder (MDD). Eur Neuropsychopharmacol. 2012;22(7):482-91. https://doi.org/10.1016/j.euroneuro.2011.11. 008.

17. Boulenger JP, Loft H, Olsen CK. Efficacy and safety of vortioxetine (Lu AA21004), 15 and $20 \mathrm{mg} /$ day: a randomized, double-blind, placebo-controlled, duloxetine-referenced study in the acute treatment of adult patients with major depressive disorder. Int Clin Psychopharmacol. 2014;29(3):138-49. https://doi. org/10.1097/YIC.0000000000000018.

18. Henigsberg N, Mahableshwarkar AR, Jacobsen P, Chen Y, Thase ME. A randomized, double-blind, placebo-controlled 8-week trial of the efficacy and tolerability of multiple doses of Lu AA21004 in adults with major depressive disorder. J Clin Psychiatry. 2012;73(7):953-9. https://doi.org/10.4088/JCP.11m07470.

19. Jain R, Mahableshwarkar AR, Jacobsen PL, Chen Y, Thase ME. A randomized, double-blind, placebo-controlled 6-wk trial of the efficacy and tolerability of $5 \mathrm{mg}$ vortioxetine in adults with major depressive disorder. Int J Neuropsychopharmacol. 2013;16(2): 313-21. https://doi.org/10.1017/S1461145712000727.

20. Alam MY, Jacobsen PL, Chen Y, Serenko M, Mahableshwarkar AR. Safety, tolerability, and efficacy of vortioxetine (Lu AA21004) in major depressive disorder: results of an open-label, flexible-dose, 52-week extension study. Int Clin Psychopharmacol. 2014;29(1): 36-44. https://doi.org/10.1097/YIC.0000000000000010.

21. Areberg J, Sogaard B, Hojer AM. The clinical pharmacokinetics of Lu AA21004 and its major metabolite in healthy young volunteers. Basic Clin Pharmacol Toxicol. 2012;111(3):198-205. https://doi.org/10.1111/j.1742-7843.2012.00886.x.

22. Lundbeck. Brintellix [EMA]. 2013.

23. Areberg J, Petersen KB, Chen G, Naik H. Population pharmacokinetic meta-analysis of vortioxetine in healthy individuals. Basic Clin Pharmacol Toxicol. 2014;115(6):552-9. https://doi. org/10.1111/bcpt.12256.

24. Nerella NG, Block LH, Noonan PK. The impact of lag time on the estimation of pharmacokinetic parameters. I. One-compartment open model. Pharm Res. 1993;10(7):1031-6.

25. Bundgaard C, Eneberg E, Sanchez C. P-glycoprotein differentially affects escitalopram, levomilnacipran, vilazodone and vortioxetine transport at the mouse blood-brain barrier in vivo. Neuropharmacology. 2016;103:104-11. https://doi.org/10.1016/j. neuropharm.2015.12.009.

26. Chen G, Lee R, Hojer AM, Buchbjerg JK, Serenko M, Zhao Z. Pharmacokinetic drug interactions involving vortioxetine $(\mathrm{Lu}$ AA21004), a multimodal antidepressant. Clin Drug Investig. 2013;33(10):727-36. https://doi.org/10.1007/s40261-013-0117-6.

27. Welling PG. Effects of food on drug absorption. Annu Rev Nutr. 1996;16:383-415. https://doi.org/10.1146/annurev.nu.16.070196. 002123.

28. Mayer M, Xie J, Serenko M. Effect of formulation and food on the pharmacokinetics of Lu AA21004 in healthy subjects. Clin Pharmacol Ther. 2012;91(Suppl. 1):S69.
29. Wang Y, Wojtkowski T, Agyemang A, Homery C, Karim A. Effect of food on the pharmacokinetics of Lu AA21004 in healthy volunteers. J Clin Pharmacol. 2009;49:1115.

30. Nilausen D, Hojer AM, Bendahl H. Absorption, metabolism, and excretion of an oral dose of $\left[{ }^{14} \mathrm{C}\right]-\mathrm{LU}$ AA2 $100450 \mathrm{mg}$ in healthy men. J Clin Pharmacol. 2009;49:1113.

31. Roberts JA, Pea F, Lipman J. The clinical relevance of plasma protein binding changes. Clin Pharmacokinet. 2013;52(1):1-8. https://doi.org/10.1007/s40262-012-0018-5.

32. Mayer M, Wu R, Serenko M, Mahableshwarkar AR. Effect of renal impairment on the pharmacokinetics of Lu AA21004. Clin Pharmacol Drug Dev. 2012;1(4):188.

33. Hvenegaard MG, Bang-Andersen B, Pedersen H, Jorgensen M, Puschl A, Dalgaard L. Identification of the cytochrome P450 and other enzymes involved in the in vitro oxidative metabolism of a novel antidepressant, Lu AA21004. Drug Metab Dispos. 2012;40(7):1357-65. https://doi.org/10.1124/dmd.112.044610.

34. Uldam HK, Juhl M, Pedersen H, Dalgaard L. Biosynthesis and identification of an $\mathrm{N}$-oxide/ $\mathrm{N}$-glucuronide metabolite and first synthesis of an $\mathrm{N}$-O-glucuronide metabolite of Lu AA21004. Drug Metab Dispos. 2011;39(12):2264-74. https://doi.org/10. 1124/dmd.111.040428.

35. Sheng Y, He Y, Huang X, Yang J, Wang K, Zheng Q. Systematic evaluation of dose proportionality studies in clinical pharmacokinetics. Curr Drug Metab. 2010;11(6):526-37.

36. Dudkowski C, Lee R, Wu R, Zhao Z, Serenko M. A phase 1 study to assess the effect of age, gender and race on the pharmacokinetics of single and multiple doses of $\mathrm{Lu}$ aa21004 in healthy subjects. Clin Pharmacol Ther. 2012;91(Suppl. 1):S69.

37. Nomikos GG, Tomori D, Zhong W, Affinito J, Palo W. Efficacy, safety, and tolerability of vortioxetine for the treatment of major depressive disorder in patients aged 55 years or older. CNS Spectr. 2017;22(4):348-62. https://doi.org/10.1017/ S1092852916000626.

38. Chen G, Nomikos GG, Affinito J, Jacobson W, Zhao Z, Wang S, et al. Single-dose pharmacokinetics of vortioxetine in subjects with mild, moderate, or severe hepatic impairment. San Diego, CA, USA: The American Society for Clinical Pharmacology and Therapeutics (ASCPT); 2016.

39. Stingl JC, Brockmoller J, Viviani R. Genetic variability of drugmetabolizing enzymes: the dual impact on psychiatric therapy and regulation of brain function. Mol Psychiatry. 2013;18(3):273-87. https://doi.org/10.1038/mp.2012.42.

40. Chen G, Nomikos GG, Affinito J, Zhao Z. Lack of effect of vortioxetine on the pharmacokinetics and pharmacodynamics of ethanol, diazepam, and lithium. Clin Pharmacokinet. 2016;55(9):1115-27. https://doi.org/10.1007/s40262-016-0389-0.

41. Chen G, Zhang W, Serenko M. Lack of effect of multiple doses of vortioxetine on the pharmacokinetics and pharmacodynamics of aspirin and warfarin. J Clin Pharmacol. 2015;55(6):671-9. https://doi.org/10.1002/jcph.456.

42. Pan L, Chen G, Sveigaard C, editors. Vortioxetine: in vitro assessment and clinical implications of transporter inhibitionbased drug interactions. In: 13th European International Society for the Study of Xenobiotics (ISSX) Annual Meeting; June 2015; Glasgow.

43. Wang Y, Wojtkowski T, Hanson E, Harris S, Karim A. An openlabel multiple-dose study in healthy adults to assess the drug interaction potential of Lu AA21004 using the Indiana cocktail. J Clin Pharmacol. 2009;49:1114.

44. Areberg J, Luntang-Jensen M, Sogaard B, Nilausen DO. Occupancy of the serotonin transporter after administration of $\mathrm{Lu}$ AA21004 and its relation to plasma concentration in healthy subjects. Basic Clin Pharmacol Toxicol. 2012;110(4):401-4. https://doi.org/10.1111/j.1742-7843.2011.00810.x. 
45. Stenkrona P, Halldin C, Lundberg J. 5-HTT and 5-HT(1A) receptor occupancy of the novel substance vortioxetine (Lu AA21004). A PET study in control subjects. Eur Neuropsychopharmacol. 2013;23(10):1190-8. https://doi.org/10.1016/j. euroneuro.2013.01.002.

46. Meyer JH, Wilson AA, Sagrati S, Hussey D, Carella A, Potter WZ, et al. Serotonin transporter occupancy of five selective serotonin reuptake inhibitors at different doses: an $\left[{ }^{11} \mathrm{C}\right] \mathrm{DASB}$ positron emission tomography study. Am J Psychiatry. 2004;161(5):826-35. https://doi.org/10.1176/appi.ajp.161.5.826.

47. Conen S, McKie S, Smallman RP, Dutta A, Dawson GR, Smith J, et al. Effects of vortioxetine on resting-state activity in subjects remitted from depression and healthy controls. Eur Neuropsychopharmacol. 2015;25(Suppl. 2):S442. https://doi.org/10.1016/ S0924-977X(15)30593-9.

48. Wang Y, Nomikos GG, Karim A, Munsaka M, Serenko M, Liosatos M, et al. Effect of vortioxetine on cardiac repolarization in healthy adult male subjects: results of a thorough QT/QTc study. Clin Pharmacol Drug Dev. 2013;2(4):298-309. https://doi. org/10.1002/cpdd.51.

49. Theunissen EL, Street D, Hojer AM, Vermeeren A, van Oers A, Ramaekers JG. A randomized trial on the acute and steady-state effects of a new antidepressant, vortioxetine (Lu AA21004), on actual driving and cognition. Clin Pharmacol Ther. 2013;93(6):493-501. https://doi.org/10.1038/clpt.2013.39.

50. Wilson S, Hojer AM, Buchberg J, Areberg J, Nutt DJ. Differentiated effects of the multimodal antidepressant vortioxetine on sleep architecture. Part 1. A pharmacokinetic/pharmacodynamic comparison with paroxetine in healthy men. J Psychopharmacol. 2015;29(10):1085-91.

https://doi.org/10.1177/ 0269881115599387.

51. Wang Y, Wojtkowski T, Devoti A, Priestly A, Karim A. Effect of Lu AA21004 on the pharmacokinetics and pharmacodynamics of ethinyl estradiol $(30 \mathrm{lg})$ and levonorgestrel $(150 \mathrm{lg})$ in healthy adult women. J Clin Pharmacol. 2009;49:1114.

52. Frank C. Recognition and treatment of serotonin syndrome. Can Fam Physician. 2008;54(7):988-92.

53. Naik H, Chan S, Vakilynejad M, Chen G, Loft H, Mahableshwarkar AR, et al. A population pharmacokinetic-pharmacodynamic metaanalysis of vortioxetine in patients with major depressive disorder. Basic Clin Pharmacol Toxicol. 2016;118(5):344-55. https://doi. org/10.1111/bcpt.12513. 